Review Article

\title{
Natural Killer Cell Dysfunction in Obese Patients with Breast Cancer: A Review of a Triad and Its Implications
}

\author{
Esraa Elaraby, ${ }^{1}$ Abdullah Imadeddin Malek, ${ }^{1}$ Hanan W. Abdullah, ${ }^{1}$ \\ Noha Mousaad Elemam $\mathbb{D}^{1,},{ }^{1,2}$ Maha Saber-Ayad, ${ }^{1,2,3}$ and Iman $M$. Talaat $\mathbb{D}^{1,2,4}$ \\ ${ }^{1}$ College of Medicine, University of Sharjah, Sharjah, UAE \\ ${ }^{2}$ Sharjah Institute for Medical Research, University of Sharjah, Sharjah, UAE \\ ${ }^{3}$ Faculty of Medicine, Cairo University, Cairo, Egypt \\ ${ }^{4}$ Faculty of Medicine, Alexandria University, Alexandria, Egypt
}

Correspondence should be addressed to Noha Mousaad Elemam; nelemam@sharjah.ac.ae and Iman M. Talaat; italaat@sharjah.ac.ae

Received 5 March 2021; Revised 4 May 2021; Accepted 19 May 2021; Published 8 June 2021

Academic Editor: Xue-Li Zhang

Copyright (c) 2021 Esraa Elaraby et al. This is an open access article distributed under the Creative Commons Attribution License, which permits unrestricted use, distribution, and reproduction in any medium, provided the original work is properly cited.

\begin{abstract}
Natural killer cells (NK cells) are a crucial constituent of the innate immune system as they mediate immunity against viruses, bacteria, parasites, and most importantly, tumor cells. The exact mechanism of how the innate immune system and specifically NK cells interact with cancer cells is complex and is yet to be understood. Several factors that constitute the tumor microenvironment (TME) such as hypoxia and TGF- $\beta$ are believed to play a role in the complex physiological reaction of NK cells to tumor cells. On the other hand, several risk factors are implicated in the development and progression of breast cancer, most importantly: obesity. Cytokines released from adipose tissue such as adipokines, leptin, and resistin, among others, are also believed to facilitate tumor progression. In this study, we aimed to build a triad of breast cancer, obesity, and NK cell dysfunction to elucidate a link between these pillars on a cellular level. Directing efforts towards solidifying the link between these factors will help in designing a targeted immunotherapy with a low side-effect profile that can revolutionize breast cancer treatment and improve survival in obese patients.
\end{abstract}

\section{Breast Cancer and Its Microenvironment}

Breast cancer is considered the most common type of cancer among women. The current global burden of breast cancer is substantial as it affected approximately 2.3 million women in 2020 alone. Moreover, breast cancer accounts for 1 in every 4 cancer cases among females and is the preeminent cause of death in women. It is estimated that 1 in 6 cancer deaths is due to breast cancer and the approximate number of deaths in 2020 was 684,996 [1]. Moreover, a wide range of risk factors related to breast cancer are reported, including postponement of childbearing, early menarche, genetic mutations, and most importantly, physical inactivity and obesity [2]. Despite the fact that breast cancer has a good prognosis if discovered at early stages, $50-80 \%$ of the cases are unfortunately discovered in later stages, making the tumor cells more resistant to therapy, hence favoring a quiet poor prognosis [3].

A number of factors influence management plans and decision-making for patients with breast cancer. These factors include tumor morphology, grade, size, metastases, and notably the expression of estrogen receptors (ER), progesterone receptors (PR), and human epidermal growth factor receptor 2 (HER2) [4-6]. Primarily, several biological subtypes of breast cancer exist and can be delineated based upon their genotypic and phenotypic features. This classification is achieved by a multitude of laboratory techniques including immunohistochemistry and genetic expression profiling. Further genetic profiling and molecular analysis of breast cancer led to its classification into several subtypes including ER+ luminal A and luminal B, HER2-enriched, and triplenegative breast cancer [7]. Histologic analysis of breast 
cancer is diagnostic and stratifies breast cancer to its subtypes. It was identified that the most common subtype is invasive ductal carcinoma, which makes up 50\%-75\% of patients. The second most common subtype is invasive lobular carcinoma, making up 5\%-15\% of patients, while mixed ductal/lobular carcinomas and other rarer histological subtypes make up the remainder of patients [8].

Interestingly, according to a cohort study conducted in the $\mathrm{UK}$, a higher body mass index (BMI) in postmenopausal women was associated with a $20-40 \%$ increased risk of breast cancer development. This led to the development of a concept known as the "obesity paradox" which implies that while morbidly high BMI was associated with a poorer prognosis, a moderately high BMI showed a better prognosis and response to therapy in premenopausal women specifically $[9,10]$.

An overview of the breast tumor microenvironment (TME) will help provide a better understanding of the role of a variety of cell types, their effects on each other, and the surrounding cells leading to the proliferation and metastasis of the tumor. There are various cell types in the breast cancer TME such as breast cancer cells, epithelial mesenchymal cells (EMTs), and stromal cells which include fibroblasts, adipocytes, endothelial cells, and immune cells. Cell adhesion is reduced between tumor cells in the TME in comparison to normal epithelium whereby cells are tightly attached to each other via cell junctions and cell adhesion molecules (CAMs). This feature of decreased adherence facilitates its dissociation and proliferation [11]. Tumor cells divide in an uncontrollable manner, which mandates different oxygen and nutrient supplies, needs to the cancerous cells at different locations resulting in a hypoxic environment that alters protein expression leading to further mutations in tumor cells and triggers angiogenesis allowing direct access to blood and lymphatic fluid for metastasis [12]. Hence, cellular mutations could result in uncontrollable cell division and hence the development of a tumor [13].

One of the cell types present in the tumor microenvironment is fibroblasts. Upon tissue insult, fibroblasts are converted into myofibroblasts that could further transform into tumor-associated fibroblasts (TAFs). TAFs play a major role in promoting tissue fibrosis, angiogenesis, immunosuppression, and metastasis. Additionally, cytokines in the TME facilitate the conversion of adipocytes into tumor-associated adipocytes (TAAs). TAAs secrete additional cytokines, adipokines, free fatty acids (FFAs), and matrix metalloproteinases (MMPs), enrolling immune cells to the TME and leading to further inflammation. Other cell types include vascular cells that constitute a monolayer of endothelial cells (ECs) that are in direct contact with the blood. ECs direct inflammatory cells towards foreign molecules and the inflammatory milieu in response to infection or inflammation [14]. Finally, immune cells are recruited to the TME, as it represents a highly inflammatory site $[15,16]$.

\section{Natural Killer Cells}

The immune system is implied in the defense against pathogens and tumor cells. Both branches of the immune system, namely, the innate and adaptive immune systems with their cellular components, soluble molecules, and cellular receptors that have different functions, are ultimately aimed at eradicating pathogens or tumor cells from the body. The innate immune system constitutes the initial and early response of the body and thus acts rapidly and in a nonspecific manner to prevent the spread of the foreign pathogen. This is achieved by a plethora of factors including but not limited to complement activation and cytotoxic molecule release as well as activation of other immune cells [17].

Natural killer cells (NK cells) are large granular lymphocytes that represent a crucial constituent of the innate immune system as they mediate immunity against viruses, bacteria, parasites, and most importantly, tumor cells [18]. NK cells are defined by the expression of an adhesion molecule CD56 and by the absence of the T cell marker CD3 [19]. Several subdivisions of NK cells exist based on the cellular expression of CD56 and the Fc (gamma) receptor CD16. The most common subdivision of NK cells is based on function, whether they primarily induce cytotoxic activity or release proinflammatory cytokines. The cytotoxic cells are mostly CD56 ${ }^{\text {dim }}$ CD $16^{\text {bright }}$ and represent about $90 \%$ of all NK cells [20]. Cytotoxicity is mediated against target cells by the secretion of cytotoxic molecules or death receptormediated apoptosis. Granzymes and perforins are cytotoxic molecules that result in cell death. Another possible method is via activation of death cell receptors such as Fas ligand and TNF-related apoptosis-inducing ligand (TRAIL), which leads to the classical caspase-mediated apoptosis. The other subtype includes CD56 ${ }^{\text {bright }} \mathrm{CD} 16^{\text {dim }}$ that has an immunoregulatory role and releases many cytokines such as IFN- $\gamma$ and TNF- $\alpha$ [21-23].

In addition, NK cell functionality is dependent and dismantled by several factors. For instance, aging is a condition that greatly impairs NK cells' function [24]. Another major factor that will be further discussed in this review is obesity. Several factors are believed to affect NK cell function in obese patients in comparison to lean patients. This formulates a triangle of interest: obesity, breast cancer, and NK cell dysfunction. In this review, we aim to further expand the link between these factors and their effect on the innate immune system in fighting breast cancer.

2.1. Mechanism of Natural Killer Cell Activation. NK cell function is tightly regulated by a repertoire of membraneexpressed inhibitory and activating receptors, which are the "nuts and bolts" of NK cell function [25]. Inhibitory receptors recognize normal healthy cells via the self-major histocompatibility complex (MHC) class I molecules and thus play a crucial role in self-tolerance. NK cell inhibitory receptors include members of the C-type lectin-like receptor, leukocyte immunoglobulin-like receptors (LILRs), and the killer immunoglobulin-like receptors (KIRs) [26, 27]. NK cells are activated when they encounter other cells that are not expressing MHC class I receptors like cancer cells or viral-infected cells. Such cells are undergoing stress which downregulates the expression of MHC class I and upregulates the expression of other molecules/ligands that further activate the NK cells. Upon this interaction, NK cells either are either directly activated and eliminate the cells by 
cytotoxicity or indirectly release their proinflammatory cytokines which will eventually result in the death of these stressed cells. The lack of MHC class I expression is insufficient for the activation of NK cells, but other NK receptors contribute to the complete activation of NK cells when they are stimulated, including NKG2D and NCRs like NKp30,NKp46, and NKp44 [28].

2.2. Natural Killer Cells and Breast Cancer. Being innate cells, NK cells can lyse tumor target cells without prior sensitization or clonal expansion, unlike T cells. NK cells play a fundamental role in cancer immunosurveillance by performing their antitumor activity $[29,30]$. This has been supported by studies where elimination of NK cells led to increased malignancy occurrence [31-33]. NK cells perform antitumor activity when the expression of MHC class I molecules is downregulated. Additionally, upregulation of stress-induced molecules such as ligands of the activating receptor $\mathrm{C}$ lectin receptor D (NKG2D) on tumor cells marks them susceptible to NK cell killing [34]. Moreover, NK cells have been found to enhance $\mathrm{T}$ cell infiltration, thus triggering immune responses through their cytokines and chemokine secretion $[35,36]$. In addition, NK cells possess antimetastatic activity by possible elimination of circulating tumor cells $[36,37]$.

The exact mechanism of how the innate immune system and specifically NK cells interact with breast cancer cells is complex and is yet to be understood. However, several factors that are released by breast cancer cells and constitute the TME are believed to contribute to this complex physiologic reaction to tumor cells. Additionally, the TME is thought to play a major role in various processes such as tumor development, progression, growth, and metastasis. Most importantly, TME mediates immune suppression leading to tumor progression by inhibiting the immune system antitumor activities $[38,39]$.

\subsection{Role of Natural Killer Cells in Tumor Microenvironment.} The protective functions of NK cells are hindered by the suppressive cytokines found in the TME. Among the cytokines released in the TME is tumor growth factor- (TGF-) $\beta$, which is released by the tumor cells, Tregs, and other stroma cells. TGF- $\beta$ is responsible for inhibiting the function of NK cells in both direct and indirect manners. Initially, TGF- $\beta$ suppresses the IFN- $\gamma$ production along with the levels of NKG2D and NKp30 on the cell surface. Moreover, TGF- $\beta$ binds to certain receptors that contain TGFBR1 and TGFBR2 subunits that will propagate the signal transduction of phosphorylating SMAD2 and SMAD3 proteins which will bind to SMAD4 resulting in a heterotrimeric transcriptional structure. The SMAD proteins are the main signal transducers for the receptors of the TGF- $\beta$ superfamily. The role of TGF- $\beta$ was not only limited to disabling NK cells but also to inducing the conversion of NK cells into NK-ILC-1, intermediate cell type (int ILC1s) which is by default a weaker cytolytic cell compared to NK cells. This will end up with a poorer cancer surveillance and eventually pave the way for further cancer evasion [40].

Metabolic derangement is one of the hallmarks characterizing the TME, leading to NK cell dysfunction. For instance, lactate level is increased in the TME and leads to suppression of cytotoxic T cells and NK cell proliferation and reduction of their cytokine production [41]. Furthermore, hypoxic environment such as that in the TME downgrades the NK cell functions by inhibiting the activating receptors such as NKG2D, NKp30, and CD16. Additionally, studies demonstrated that the depletion of NK cells prior to implantation of tumor cells in mice was found to be associated with a more aggressive picture of tumor metastasis [42-44].

An emerging role of NK cells in targeting tumor cells is being recognized by their action on cancer stem cells. Cancer stem cells (CSCs) are undifferenced cells involved in the growth of tumors. They are characterized by an expression profile consisting of low levels of CD54 and PD-1 and high expression of CD44. This profile increases the susceptibility of CSC to be targeted by NK cells but conversely induces their resistance to chemotherapy. NK cells drive the CSCs to differentiate in a way where the expression of MHC-1, CD54, and PD-L1 is elevated, resulting in stunted tumor growth and decreased metastasis. This delineates the importance of NK cells not only in restraining tumors but also in limiting their growth. On the contrary, other studies demonstrated that the CSCs in breast tumors are resistant to any NK cell activity and thus, more research is needed to further elucidate and establish the true link between NK cells and CSCs of breast cancer [45].

\section{Obesity}

Obesity can be defined as the excessive and abnormal accumulation of adipose tissue and is commonly classified based on the body mass index (BMI). The BMI of an individual can be calculated by dividing the body weight in kilograms by the height in meters squared $\left(\mathrm{kg} / \mathrm{m}^{2}\right)[46,47]$.

Obesity has come to light as a major public health problem that leads to approximately 4 million deaths and 120 million disability-adjusted life-years (DALY). According to the WHO, obesity has tripled on a global scale since 1975. In 2016, 1.9 billion people at 18 or more were overweight or obese. Unluckily, obesity rates in the MENA region are not updated, but the indices show that the number of obese people is escalating. This could be attributed to the higher levels of urbanization and technical advancements in the MENA region that contribute to a sedentary lifestyle and unhealthy food options [48]. Most of the subsequent complications caused by obesity share a common feature which is a state of subclinical chronic inflammation that is a crucial component of tumor development and progression [49].

Being obese or overweight is a well-known risk factor for the development of several chronic health disorders such as type 2 diabetes and cardiovascular diseases, among many others. Along with obesity comes an increased susceptibility to infection and decreased ability to fight off infections efficiently. Most importantly, there is an increase in the incidence of several types of cancers, e.g., colorectal, endometrial, pancreatic, and breast cancer, with an increased incidence and poorer prognosis in obese patients. In fact, $14-20 \%$ of the cancers have been attributed to obesity [50]. 
Consequently, obesity results in the increase in the accumulation of adipose tissue mass. Adipose tissue, in lean state, acts as an energy-storage reservoir and the largest endocrine organ that is thought to secrete a multitude of adipokines including but not limited to leptin, adiponectin, resistin, and estrogens as well as interleukin-6 (IL-6), all of which orchestrate a variety of reactions in the body. On the contrary, examples of anti-inflammatory and adipose-resident immune cells are Tregs, eosinophils, T-helper 2 cells, and M2 macrophages [51]. Adipocytes are the prominent producers of leptin in the body, where leptin acts a stimulator of multiple proinflammatory reactions and the production of IL-1, IL-6, IL-12, TNF- $\alpha$, COX2, and nitric oxide (NO). Leptin levels are found to be higher in obese patients in comparison with lean patients contributing to the chronic inflammation that occurs in obesity. In fact, a study suggested that levels of leptin could be used to predict type, grade, prognosis, and recurrence in breast cancer based on its immunohistochemical staining $[15,52]$. It is worth mentioning that the expansion of adipocytes to meet the increased energy storage demands could eventually cause these cells to become apoptotic, thus attracting proinflammatory macrophages and forming crown-like structures: a hallmark of the inflammatory environment in adipose tissue [53].

3.1. Breast Cancer and Obesity. Even though inherited genetic factors such as BRCA1/2 mutations result in 5-10\% of cases of breast cancer, lifestyle is now considered as an increasingly contributing factor to the etiology of breast cancer [54]. The incidence of breast cancer recurrence and mortality rate increases with obesity due to the dysregulation of a variety of biological and nonbiological factors. These factors include advanced stages of breast cancer presentation, increased risk of second primary cancer (i.e., primary cancer in other tissues), and the use of a suboptimal level of chemotherapeutic agents compared to the relative body size [55].

There is an aberration involving multiple molecular pathways involving adipokines, endogenous sexual hormonal levels, and most importantly, inflammation [56]. Out of these molecular pathways, there is an abnormal regulation in the levels of estrogen due to the aromatization of the adipose tissue. Inflammatory cytokines are thus recruited such as TNF- $\alpha$, IL- 6 , and prostaglandin E2 adipokines, not to mention oxidative stress, which contributes to carcinogenesis. The molecular factors induce intracellular interference which activate mitogen protein kinase (MAPK) and phosphatidylilinositol-3-phosphate/mammalian target of rapamycin (mTOR pathway), which play a role in the progression of cell cycle and protein synthesis. In breast cancer, the associated genes of obesity collectively lead to an increase in fat mass and production of cytokines such as leptin, which has been associated with a higher risk of cancer development [56].

In addition, the association of breast cancer with adiposity has been linked to higher energy states that may enhance tumor growth by providing an increased level of ATP for inducing cell growth and replication. There is evidence for a "metabolic threshold" in promoting breast cancer, which was supported by the development of targeted metabolic inhibitors as cancer therapeutics $[57,58]$.
3.2. Natural Killer Cells and Obesity. There is evidence that points towards a decrease in the number of NK cells in the blood and tissues of obese individuals. Furthermore, there is a decrease in the number of NK cells in the blood and organs of obese rats [59-62]. Other data reveal no change in the number of NK cells or even an increase in the NK cells present in the blood and tissues of obese individuals [60-66]. This can be attributed to several various factors including but not limited to metabolic differences between species and strains as well as discrepancies between the development and migration processes of NK cells in different species [67]. In addition, such discrepancies could be due to several reasons including the choice of markers and quantitative methods used for NK cells as well as differences in the study population such as BMI, gender, ethnicity, body composition, and variance $[68,69]$.

From a functional prospective, data in obese individuals revealed a clear decrease in the activating receptors on NK cells, namely, NKp46, as well as TRAIL, functional markers of NK cells [60]. On the other hand, other studies revealed a highly activated status of NK cells in obese individuals, as highlighted by an increase in the expression of CD69 and NKp46 as well as PD-1, while there was a decline in the inhibiting complex NKG2A/CD94, thus indicating an activated status of NK cells [70]. While NK cells seemed to be increasingly activated, their functionality was greatly impaired as evidenced by a decreased secretion of mediators necessarily for their function such as granzyme B, perforin, and macrophage inflammatory protein $\beta$. This decline in functionality could be attributed to exhaustion of NK cells which occurs faster in obese individuals compared to normal-weight individuals $[71,72]$. To further confirm findings on the effect of obesity on NK cell function, some studies interestingly demonstrated that the impaired NK cell function can be restored and normalized following the loss of body weight and fat mass in obese individuals. This includes an increase in the CD69 levels and granzyme B secretion as well as a decrease in IFN- $\gamma$ production [73]. On the other hand, several studies associated the caloric restriction with improved NK cell cytotoxicity and elevated expression of activation markers CD69, TNF- $\alpha$, and GM-CSF. However, few studies showed a decrease in NK cell numbers and cytotoxicity upon weight reduction [59].

Interestingly, chronic low-grade inflammation of fat tissue can be appreciated by an increase in immune cells such as macrophages, T cells, and NK cells [74]. Studies show varying data with regard to NK cells in adipose tissue. Some studies reported an increase in NK cells in adipose tissue of obese individuals, while others demonstrate a decreased or no change in the number of NK cells between obese and lean individuals [74-78]. However, a shift from the cytotoxic CD56 ${ }^{\text {dim }}$ subpopulation of NK cells to the CD56 ${ }^{\text {bright }}$ cytokine secreting subset was observed in obese individuals, which serves to explain the reason for increased cytokine secretion in obese individuals [79]. In mice, an increased level of activating receptor NKp46 on adipose tissues seemed to increase the proliferation of NK cells that secrete IFN- $\gamma$, which eventually leads to a polarization in macrophages to the M1 proinflammatory macrophages [74]. A decreased 
expression of NK cells activating receptors such as NKp30 and NKp44 was observed in adipose tissue of obese individuals, which might be a contributing factor to the increased risk of cancer development in obese individuals as well as increased susceptibility to infection [79].

In obesity, there are higher levels of free fatty acids (FFAs) circulating freely all over the body. NK cells have shown to absorb these FFAs and accumulate lipid droplets. Interestingly, NK cells that accumulated more lipid droplets had merely zero perforin and granzyme levels as detected by flow cytometry, resulting in impaired NK cell cytotoxicity against cancer cells and hence helping the tumor cells to further grow and metastasize. Lipid-rich environment was proven to disturb the mTORC1 pathway in NK cells, a pathway that plays a prominent role in the NK cell function and IFN- $\gamma$ production [80]. On the other hand, obesity could be a stimulator of the peroxisome proliferator-activated receptor PPAR $\alpha / \delta$ target genes in NK cells that encourage the NK cells to further accumulate more lipids and hence hinder the cytotoxicity of NK cells [81]. NK cells residing in the visceral adipose tissue (VAT) are activated due to the increased stimulation of NCR-1 signaling by the surrounding adipocytes. As a result, IFN- $\gamma$ production is elevated which elicits M1 polarization in the macrophages residing in the adipose tissue. Moreover, M1 macrophages have a pivotal role in inducing further inflammation, further contributing to insulin resistance and obesity. This was further proved by a study where the total number of macrophages and specifically M1 macrophages was lower in NK-depleted mice compared to normal mice fed with a high-fat diet (HFD) [74].

Moreover, NK cell functions and cytotoxicity were seen to improve following restriction of energy intake and lowfat diet [82-84]. Most studies demonstrate a stimulating effect of caloric restriction on NK cell function, while others have demonstrated opposite findings with reduced killing and impaired maturation of NK cells as well as decreased cytokine production $[85,86]$. Lack of weight cycling was associated with higher $\mathrm{NK}$ cell activity, demonstrating improved NK cell function with lack of weight gain and weight loss [87]. Similar findings were seen with bariatric surgery effects with contradicting findings [88, 89].

Another possible link between NK cells, obesity, and cancer was highlighted in the study by Mariani et al. This study showed a reduced NK cell number in the colon tissue of obese patients in comparison to normal-weight patients. Hence, this could be a contributing factor to the increased risk of colon cancer in obese individuals [84].

\section{Natural Killer Cell-Mediated Immunotherapeutics in Breast Cancer}

As noted from the previous sections, NK cells are unique in exerting an innate immune activity that is antigen independent. They represent an excellent companion to immunotherapy in clinical settings by having the ability to distinguish "self" from "missing-self." Over the last few years, many studies showed NK cells as promising effectors in tumor therapy [90]. There is mounting evidence of the potential use of NK cells as a therapeutic tool in clinical practice.

There are still many open research questions related to NK cell metabolism modification in a trial to enhance the survival and activity of those key promising cells in the TME of solid tumors, including breast cancer. Several metabolic modulation strategies were investigated to support the NK cell survival and activity in the TME. Naturally, NK cells survive for 2 weeks $[18,20]$, but the infusion of IL-2 and/or IL-15 showed advantageous effects on the NK cell survival (as in the context of adoptive transfer therapy in patients with acute myeloid leukemia) [91]. Another study by Liu et al. showed that IL-15 production by transduced cord blood NK cells critically improved their function [92]. These strategies are likely to improve delivering this therapy in the clinical setting and to overcome a major limitation to current CAR-T cell therapies. It should be noted that defining a metabolic pathway for the NK cells is a step towards identifying a therapeutic target that addresses this pathway and specifically activates the cytotoxic activity of the NK cells. Production of certain metabolites limits the survival and function of NK cells, including pyruvate dehydrogenase kinase 1, lactate dehydrogenase A (LDHA), and adenosine (ADO) [93]. Interestingly, lipid-lowering drugs represent a potential therapy for patients with ER-positive breast cancer. In contrast, a recent study by Qin et al. on liver-tumor-bearing murine model suggested that cholesterol accumulation in NK cells enhances their antitumor ability through increasing the formation of lipid rafts [94]. The results of the latter study reflect the diverse function of lipid metabolism in different cancers. In addition, direct activation of the citrate-malate shuttle was demonstrated to enhance glucose metabolism and hence NK cell cytotoxicity and may have a role in their persistence/survival [95]. Additionally, a study on CD8+ T cells reported that cell activation under the effect of 2-deoxy-glucose (2DG) inhibitor enhanced the generation of memory cells and antitumor functionality, which could be applied to NK cells as well [96].

On the other hand, activation of the transcription factor SREBP (sterol regulatory element-binding protein) and its control of glucose and lipid metabolism were found to be essential for the function of NK cells. Furthermore, SREBP was reported to be essential for activated NK cells, as it provides metabolic reprogramming [97]. A study by $\mathrm{Wu}$ et al. showed that SREBP inhibitors such as 27-hydroxycholesterol (27HC), accumulating in the TME, partly affect SREBPrelated glycolysis in ER-positive BC [98]. Interestingly, Baek et al. reported that treating mice submitted to highcholesterol feed with an inhibitor of CYP27A1, an enzyme important in $27 \mathrm{HC}$ biosynthesis, clearly decreases the number of metastases in mice and reverses the immune suppressive environment [99].

\section{The Triad: Effect of Obesity on Natural Killer Cell Functions in Breast Cancer}

Glucose and lipid metabolism is generally highly activated in breast cancer. This was demonstrated by several metabolomic studies of breast cancer specifically revealing enhanced 


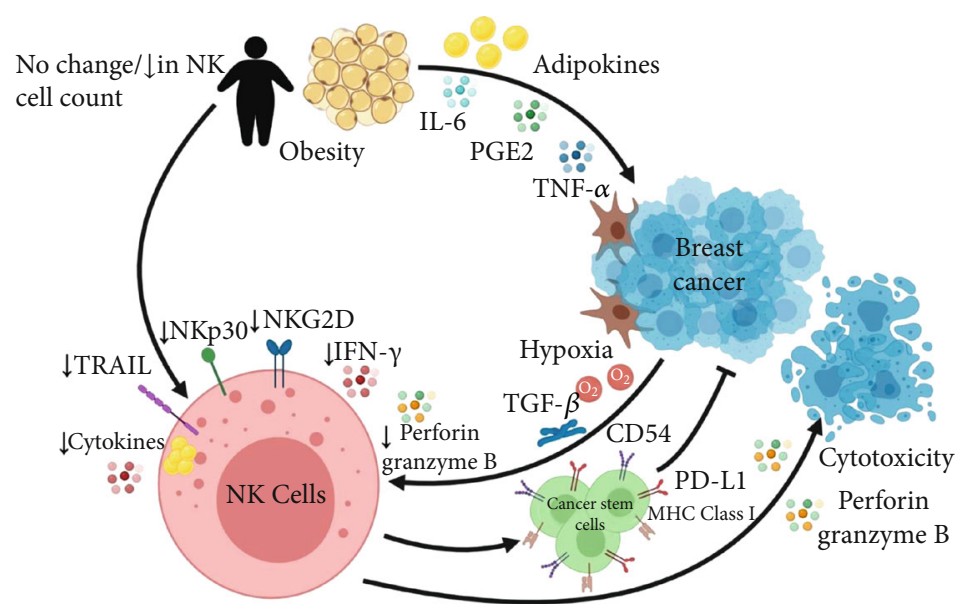

Figure 1: Triad of obesity, natural killer cells, and breast cancer. Typically, natural killer (NK) cells release perforin and granzyme B, molecules that cause cytotoxicity and induce apoptosis of breast cancer (BC) cells. Additionally, NK cells stimulate the expression of CD54, PD-L1, and MHC class I molecules on cancer stem cells, which inhibit the metastasis and proliferation of breast cancer cells. In turn, hypoxic environment of breast cancer cells and secreted TGF- $\beta$ by stromal cells in the tumor microenvironment (TME) cause a reduction in the activating receptors (NKG2D and NKp30) as well as the NK cell function: IFN- $\gamma$ production and cytotoxicity via perforin and granzyme B release. Similarly, adipose tissue causes a reduction in TRAIL, NKp30, and NKG2D expression in obese patients. Lipid droplet accumulation in NK cells leads to a reduction in cytokine release such as IFN- $\gamma$. Controversial data were reported regarding the count and status of NK cells in obese individuals. On the other arm, adipose tissue secretes IL-6, PGE2, TNF- $\alpha$, and adipokines such as leptin which trigger the proliferation of breast cancer cells via the MAPK and mTOR pathways.

fatty acid synthase and glycolysis. Obesity exerts a status of NK cell immune paralysis by affecting the NK cell metabolism and trafficking in the TME [81]. Previous reports showed that metabolic reprogramming of NK cells in obesity limits the antitumor responses through different mechanisms, mainly through $\operatorname{PPAR} \alpha / \delta$ pathway and inhibition of mTOR-mediated glycolysis [81]. Michelet et al. showed a plausible mechanistic effect of obesity that enhances lipid accumulation in NK cells through a peroxisome proliferator-activated receptor (PPAR), leading to inhibition of the mechanistic target of rapamycin(mTOR-) mediated glycolysis in NK cells. In obesity, PPAR $\alpha / \delta$ target genes are highly upregulated, thus causing inhibition of IFN- $\gamma$ production as well as the downstream transcription of other cytotoxic granules in adipose tissue NK cells [81]. This illustrates the inhibitory effect of obesity on NK cell function, which in turn contributes to further growth and metastasis of tumor cells. It is important to note, however, that NK cell function can also be impeded by breast cancer cells through the aforementioned factors such as hypoxia and increased production of TGF- $\beta$ which lead to decreased perforin and granzyme B secretion from NK cells, hence reducing its antitumor activity (Figure 1). Collectively, both breast cancer and obesity lead to enhanced breast cancer proliferation through distinct but directly related pathways, thus promoting tumor growth and metastasis.

\section{Conclusions}

In this review, we aimed to investigate the triad: obesity, breast cancer, and NK cells, to aid in the understanding of the various effects of these players in breast cancer development and progression. Extensive research is still ongoing to pin down the biomarkers associated with various types of breast cancer that impede NK cell function. Targeting these factors will help in designing targeted immunotherapy with a low side-effect profile [100]. Despite the fact that NK cells are short-lived and targeting them might not help in having a prolonged anti-inflammatory reaction against tumor cells, ongoing research is still trying to obtain features of immunological memory which result in increased NK cell survival and efficacy $[101,102]$. More studies are required to solidify the link between obesity, breast cancer, and NK cells and to identify other factors that may play a role in their interaction.

\section{Abbreviations}

\section{HC: 27-Hydroxycholesterol}

2DG: 2-Deoxy-glucose

ADO: Adenosine

BMI: $\quad$ Body mass index

CAMs: Cell adhesion molecules

DALY: Disability-adjusted life-years

ECs: Endothelial cells

EMTs: Epithelial mesenchymal cells

ER: $\quad$ Estrogen receptors

FFAs: Free fatty acids

HER2: Epidermal growth factor receptor 2

HFD: High-fat diet

KIRs: Killer immunoglobulin-like receptors

LDHA: Lactate dehydrogenase A

LILRs: Leukocyte immunoglobulin-like receptors

MAPK: Mitogen-protein kinase

MMPs: Matrix metalloproteinases

mTOR: Phosphatidylilinositol-3-phosphate/mammalian target of rapamycin

NK cells: Natural killer cells 
NKG2D: C-type lectin receptor D

NO: $\quad$ Nitric oxide

PPAR: Peroxisome proliferator-activated receptor

PR: $\quad$ Progesterone receptor

SREBP: Sterol regulatory element-binding protein

TAA: Tumor-associated adipocytes

TGF: Tumor growth factor

TME: Tumor microenvironment

TNF: Tumor necrosis factor

TRAIL: TNF-related apoptosis-inducing ligand

VAT: Visceral adipose tissue.

\section{Disclosure}

The funders had no role in study design, data collection and analysis, decision to publish, or preparation of the manuscript.

\section{Conflicts of Interest}

The authors declare no conflict of interest.

\section{Authors' Contributions}

I.T., N.M.E., A.I.M., and E.E. were responsible for the conceptualization. E.E. and A.I.M. were responsible for the resources. E.E.., A.I.M., N.M.E., H.W.A., and M.S. were responsible for writing-original draft preparation. I.T., N.M.E., E.E., A.I.M., H.W.A., and M.S. were responsible for writing, review, and editing. I.T. was responsible for funding acquisition. All authors have read and agreed to the published version of the manuscript. Esraa Elaraby and Abdullah Malek contributed equally to this work.

\section{Acknowledgments}

This work was supported by the College of Research and Graduate Studies, University of Sharjah, UAE (grant number 1901090255).

\section{References}

[1] H. Sung, J. Ferlay, R. L. Siegel et al., "Global cancer statistics 2020: GLOBOCAN estimates of incidence and mortality worldwide for 36 cancers in 185 countries," CA: A Cancer Journal for Clinicians, vol. 71, no. 3, pp. 209-249, 2021.

[2] M. Kamińska, T. Ciszewski, K. Łopacka-Szatan, P. Miotła, and E. Starosławska, "Breast cancer risk factors," Przeglad menopauzalny $=$ Menopause review, vol. 14, no. 3, pp. 196202, 2015.

[3] A. M. Gonzalez-Angulo, F. Morales-Vasquez, and G. N. Hortobagyi, "Overview of resistance to systemic therapy in patients with breast cancer," Advances in Experimental Medicine and Biology, vol. 608, pp. 1-22, 2007.

[4] T. O. Nielsen, F. D. Hsu, K. Jensen et al., "Immunohistochemical and clinical characterization of the basal-like subtype of invasive breast carcinoma," Clinical Cancer Research, vol. 10, no. 16, pp. 5367-5374, 2004.

[5] C. M. Perou, T. Sørlie, M. B. Eisen et al., "Molecular portraits of human breast tumours," Nature, vol. 406, no. 6797, pp. 747-752, 2000.
[6] T. Sørlie, C. M. Perou, R. Tibshirani et al., "Gene expression patterns of breast carcinomas distinguish tumor subclasses with clinical implications," Proceedings of the National Academy of Sciences of the United States of America, vol. 98, no. 19, pp. 10869-10874, 2001.

[7] S. M. Fragomeni, A. Sciallis, and J. S. Jeruss, "Molecular subtypes and local-regional control of breast Cancer," Surgical Oncology Clinics of North America, vol. 27, no. 1, pp. 95120, 2018.

[8] D. Dillon, A. Guidi, and S. Schnitt, "Pathology of invasive breast cancer," in Diseases of the Breast, Wolters Kluwer health, Philadelphia, PA, 5th edition, 2014.

[9] K. Bhaskaran, I. Douglas, H. Forbes, I. dos- Santos-Silva, D. A. Leon, and L. Smeeth, "Body-mass index and risk of 22 specific cancers: a population-based cohort study of $5 \cdot 24$ million UK adults," Lancet, vol. 384, no. 9945, pp. 755-765, 2014.

[10] H. Lennon, M. Sperrin, E. Badrick, and A. G. Renehan, "The obesity paradox in cancer: a review," Current Oncology Reports, vol. 18, no. 9, pp. 56-56, 2016.

[11] C. Wai Wong, D. E. Dye, and D. R. Coombe, "The role of immunoglobulin superfamily cell adhesion molecules in cancer metastasis," International journal of cell biology, vol. 2012, Article ID 340296, 9 pages, 2012.

[12] B. Muz, P. de la Puente, F. Azab, and A. K. Azab, "The role of hypoxia in cancer progression, angiogenesis, metastasis, and resistance to therapy," Hypoxia, vol. 3, pp. 83-92, 2015.

[13] B. Arneth, "Tumor microenvironment," Medicina, vol. 56, no. 1, p. 15, 2019.

[14] A. Krüger-Genge, A. Blocki, R. P. Franke, and F. Jung, "Vascular endothelial cell biology: an update," International Journal of Molecular Sciences, vol. 20, no. 18, p. 4411, 2019.

[15] G. Cildir, S. C. Akıncilar, and V. Tergaonkar, "Chronic adipose tissue inflammation: all immune cells on the stage," Trends in Molecular Medicine, vol. 19, no. 8, pp. 487-500, 2013.

[16] M. Luo, M. Brooks, and M. S. Wicha, "Epithelial-mesenchymal plasticity of breast cancer stem cells: implications for metastasis and therapeutic resistance," Current Pharmaceutical Design, vol. 21, no. 10, pp. 1301-1310, 2015.

[17] B. Alberts, A. Johnson, J. Lewis, M. Raff, K. Roberts, and P. Walter, "Innate immunity," in Molecular Biology of the Cell, Garland Science, New York, 4th edition, 2002.

[18] E. Vivier, E. Tomasello, M. Baratin, T. Walzer, and S. Ugolini, "Functions of natural killer cells," Nature Immunology, vol. 9, no. 5, pp. 503-510, 2008.

[19] L. L. Lanier, R. Testi, J. Bindl, and J. H. Phillips, "Identity of Leu-19 (CD56) leukocyte differentiation antigen and neural cell adhesion molecule," Journal of Experimental Medicine, vol. 169, no. 6, pp. 2233-2238, 1989.

[20] M. A. Cooper, T. A. Fehniger, and M. A. Caligiuri, "The biology of human natural killer-cell subsets," Trends in Immunology, vol. 22, no. 11, pp. 633-640, 2001.

[21] C. Fauriat, E. O. Long, H. G. Ljunggren, and Y. T. Bryceson, "Regulation of human NK-cell cytokine and chemokine production by target cell recognition," Blood, vol. 115, no. 11, pp. 2167-2176, 2010.

[22] B. E. Freeman, H. P. Raué, A. B. Hill, and M. K. Slifka, "Cytokine-mediated activation of NK cells during viral infection," Journal of Virology, vol. 89, no. 15, pp. 79227931, 2015. 
[23] Y. Zhang and B. Huang, "The development and diversity of ILCs, NK cells and their relevance in health and diseases," in Regulation of Inflammatory Signaling in Health and Disease, D. Xu, Ed., pp. 225-244, Springer Singapore, Singapore, 2017.

[24] J. Hazeldine and J. M. Lord, "The impact of ageing on natural killer cell function and potential consequences for health in older adults," Ageing Research Reviews, vol. 12, no. 4, pp. 1069-1078, 2013.

[25] L. L. Lanier, "Up on the tightrope: natural killer cell activation and inhibition," Nature Immunology, vol. 9, no. 5, pp. 495$502,2008$.

[26] P. Parham, "Influence of KIR diversity on human immunity," in Mechanisms of Lymphocyte Activation and Immune Regulation X, Springer US, Boston, MA, 2005.

[27] V. M. Braud, D. S. Allan, C. A. O'Callaghan et al., "HLA-E binds to natural killer cell receptors CD94/NKG2A, B and C," Nature, vol. 391, no. 6669, pp. 795-799, 1998.

[28] S. Paul and G. Lal, "The molecular mechanism of natural killer cells function and its importance in cancer immunotherapy," Frontiers in Immunology, vol. 8, pp. 1124-1124, 2017.

[29] G. Sconocchia, S. Eppenberger, G. C. Spagnoli et al., "NK cells and $\mathrm{T}$ cells cooperate during the clinical course of colorectal cancer," OncoImmunology, vol. 3, no. 8, article e952197, 2014.

[30] S. Paul, N. Kulkarni, Shilpi, and G. Lal, "Intratumoral natural killer cells show reduced effector and cytolytic properties and control the differentiation of effector Th1 cells," OncoImmunology, vol. 5, no. 12, article e1235106, 2016.

[31] M. J. Smyth, N. Y. Crowe, and D. I. Godfrey, "NK cells and NKT cells collaborate in host protection from methylcholanthreneinduced fibrosarcoma," International Immunology, vol. 13, no. 4, pp. 459-463, 2001.

[32] T. O’Sullivan, R. Saddawi-Konefka, W. Vermi et al., "Cancer immunoediting by the innate immune system in the absence of adaptive immunity," Journal of Experimental Medicine, vol. 209, no. 10, pp. 1869-1882, 2012.

[33] J. S. Orange, "Natural killer cell deficiency," The Journal of Allergy and Clinical Immunology, vol. 132, no. 3, pp. 515525, 2013.

[34] A. Mandal and C. Viswanathan, "Natural killer cells: in health and disease," Hematology/Oncology and Stem Cell Therapy, vol. 8, no. 2, pp. 47-55, 2015.

[35] M. Gooden, M. Lampen, E. S. Jordanova et al., "HLA-E expression by gynecological cancers restrains tumorinfiltrating CD8\&lt;sup\&gt;+\&lt;/sup\&gt; T lymphocytes," Proceedings of the National Academy of Sciences, vol. 108, no. 26, pp. 10656-10661, 2011.

[36] K.-J. Malmberg, M. Carlsten, A. Björklund, E. Sohlberg, Y. T. Bryceson, and H. G. Ljunggren, "Natural killer cell-mediated immunosurveillance of human cancer," Seminars in Immunology, vol. 31, pp. 20-29, 2017.

[37] A. López-Soto, S. Gonzalez, M. J. Smyth, and L. Galluzzi, "Control of metastasis by NK cells," Cancer Cell, vol. 32, no. 2, pp. 135-154, 2017.

[38] A. Poli, T. Michel, M. Thérésine, E. Andrès, F. Hentges, and J. Zimmer, "CD56bright natural killer (NK) cells: an important NK cell subset," Immunology, vol. 126, no. 4, pp. 458465, 2009.

[39] H. Gonzalez, C. Hagerling, and Z. Werb, "Roles of the immune system in cancer: from tumor initiation to metasta- tic progression," Genes \& Development, vol. 32, no. 19-20, pp. 1267-1284, 2018.

[40] Y. Gao, F. Souza-Fonseca-Guimaraes, T. Bald et al., "Tumor immunoevasion by the conversion of effector NK cells into type 1 innate lymphoid cells," Nature Immunology, vol. 18, no. 9, pp. 1004-1015, 2017.

[41] K. Fischer, P. Hoffmann, S. Voelkl et al., "Inhibitory effect of tumor cell-derived lactic acid on human T cells," Blood, vol. 109, no. 9, pp. 3812-3819, 2007.

[42] A. Diefenbach, E. R. Jensen, A. M. Jamieson, and D. H. Raulet, "Rae1 and H60 ligands of the NKG2D receptor stimulate tumour immunity," Nature, vol. 413, no. 6852, pp. 165-171, 2001.

[43] S. Malladi, D. G. Macalinao, X. Jin et al., "Metastatic latency and immune evasion through autocrine inhibition of WNT," Cell, vol. 165, no. 1, pp. 45-60, 2016.

[44] A. Spiegel, M. W. Brooks, S. Houshyar et al., "Neutrophils suppress intraluminal NK cell-mediated tumor cell clearance and enhance extravasation of disseminated carcinoma cells," Cancer Discovery, vol. 6, no. 6, pp. 630-649, 2016.

[45] K. Kaur, M. P. Nanut, M. W. Ko, T. Safaie, J. Kos, and A. Jewett, "Natural killer cells target and differentiate cancer stem-like cells/undifferentiated tumors: strategies to optimize their growth and expansion for effective cancer immunotherapy," Current Opinion in Immunology, vol. 51, pp. 170-180, 2018 .

[46] T. Pischon, U. Nöthlings, and H. Boeing, "Obesity and cancer," The Proceedings of the Nutrition Society, vol. 67, no. 2, pp. 128-145, 2008.

[47] Expert, Panel, "Executive summary of the clinical guidelines on the identification, evaluation, and treatment of overweight and obesity in adults," Archives of Internal Medicine, vol. 158, no. 17, pp. 1855-1867, 1998.

[48] S. Alnohair, "Obesity in gulf countries," International Journal of Health Sciences, vol. 8, no. 1, pp. 79-83, 2014.

[49] N. M. Iyengar, A. Gucalp, A. J. Dannenberg, and C. A. Hudis, "Obesity and cancer mechanisms: tumor microenvironment and inflammation," Journal of Clinical Oncology : Official Journal of the American Society of Clinical Oncology, vol. 34, no. 35, pp. 4270-4276, 2016.

[50] B. B. Aggarwal, R. V. Vijayalekshmi, and B. Sung, "Targeting inflammatory pathways for prevention and therapy of cancer: short-term friend, long-term foe," Clinical Cancer Research, vol. 15, no. 2, pp. 425-430, 2009.

[51] B. Antuna-Puente, B. Feve, S. Fellahi, and J. P. Bastard, "Adipokines : le chainon manquant entre insulinoresistance et obesite," Diabetes \& Metabolism, vol. 34, no. 1, pp. 2-11, 2008 .

[52] U. J. Jung and M.-S. Choi, "Obesity and its metabolic complications: the role of adipokines and the relationship between obesity, inflammation, insulin resistance, dyslipidemia and nonalcoholic fatty liver disease," International Journal of Molecular Sciences, vol. 15, no. 4, pp. 61846223,2014

[53] G. Martinez-Santibañez, K. W. Cho, and C. N. Lumeng, "Imaging white adipose tissue with confocal microscopy," Methods in Enzymology, vol. 537, pp. 17-30, 2014.

[54] L. Garcia-Estevez and G. Moreno-Bueno, "Updating the role of obesity and cholesterol in breast cancer," Breast cancer research : BCR, vol. 21, no. 1, pp. 35-35, 2019. 
[55] C. C. Wee, E. P. McCarthy, R. B. Davis, and R. S. Phillips, "Obesity and breast cancer screening," Journal of General Internal Medicine, vol. 19, no. 4, pp. 324-331, 2004.

[56] V. Simone, M. D'Avenia, A. Argentiero et al., "Obesity and breast cancer: molecular interconnections and potential clinical applications," The Oncologist, vol. 21, no. 4, pp. 404-417, 2016.

[57] J. Huang, L. Li, J. Lian et al., "Tumor-induced hyperlipidemia contributes to tumor growth," Cell Reports, vol. 15, no. 2, pp. 336-348, 2016.

[58] K. Sun, C. M. Kusminski, and P. E. Scherer, "Adipose tissue remodeling and obesity," The Journal of Clinical Investigation, vol. 121, no. 6, pp. 2094-2101, 2011.

[59] I. Bähr, J. Spielmann, D. Quandt, and H. Kielstein, “Obesityassociated alterations of natural killer cells and immunosurveillance of cancer," Frontiers in Immunology, vol. 11, pp. 245-245, 2020.

[60] I. Bähr, V. Goritz, H. Doberstein et al., "Diet-induced obesity is associated with an impaired NK cell function and an increased colon cancer incidence," Journal of Nutrition and Metabolism, vol. 2017, Article ID 4297025, 14 pages, 2017.

[61] K. Kaur, H. H. Chang, P. Topchyan et al., "Deficiencies in natural killer cell numbers, expansion, and function at the pre-neoplastic stage of pancreatic cancer by KRAS mutation in the pancreas of obese mice," Frontiers in Immunology, vol. 9, p. 1229, 2018.

[62] B. Lamas, R. Nachat-Kappes, N. Goncalves-Mendes et al., "Dietary fat without body weight gain increases in vivo MCF-7 human breast cancer cell growth and decreases natural killer cell cytotoxicity," Molecular Carcinogenesis, vol. 54, no. 1, pp. 58-71, 2015.

[63] H. Nave, G. Mueller, B. Siegmund et al., "Resistance of Janus kinase-2 dependent leptin signaling in natural killer (NK) cells: a novel mechanism of NK cell dysfunction in dietinduced obesity," Endocrinology, vol. 149, no. 7, pp. 33703378,2008

[64] P. Haas, R. H. Straub, S. Bedoui, and H. Nave, "Peripheral but not central leptin treatment increases numbers of circulating NK cells, granulocytes and specific monocyte subpopulations in non- endotoxaemic lean and obese LEW-rats," Regulatory Peptides, vol. 151, no. 1-3, pp. 26-34, 2008.

[65] N. G. Barra, I. Y. Fan, J. B. Gillen et al., "High intensity interval training increases natural killer cell number and function in obese breast cancer-challenged mice and obese women," Journal of cancer prevention, vol. 22, no. 4, pp. 260-266, 2017.

[66] P. Behrendt, T. Buchenauer, R. Horn et al., "Diet-induced obesity, exogenous leptin-, and MADB106 tumor cell challenge affect tissue leukocyte distribution and serum levels of cytokines in F344 rats," Endocrine, vol. 38, no. 1, pp. 104$112,2010$.

[67] I. Bähr, J. Jahn, A. Zipprich, I. Pahlow, J. Spielmann, and H. Kielstein, "Impaired natural killer cell subset phenotypes in human obesity," Immunologic Research, vol. 66, no. 2, pp. 234-244, 2018.

[68] B. Lamas, N. Goncalves-Mendes, R. Nachat-Kappes et al., "Leptin modulates dose-dependently the metabolic and cytolytic activities of NK-92 cells," Journal of Cellular Physiology, vol. 228, no. 6, pp. 1202-1209, 2013.

[69] C. D. Wrann, T. Laue, L. Hübner et al., "Short-term and longterm leptin exposure differentially affect human natural killer cell immune functions," American Journal of Physiology.
Endocrinology and Metabolism, vol. 302, no. 1, pp. E108E116, 2012.

[70] J. H. Gong, G. Maki, and H. G. Klingemann, "Characterization of a human cell line (NK-92) with phenotypical and functional characteristics of activated natural killer cells," Leukemia, vol. 8, no. 4, pp. 652-658, 1994.

[71] L. M. Tobin, M. Mavinkurve, E. Carolan et al., "NK cells in childhood obesity are activated, metabolically stressed, and functionally deficient," JCI Insight, vol. 2, no. 24, 2017.

[72] S. Viel, L. Besson, E. Charrier et al., "Alteration of natural killer cell phenotype and function in obese individuals," Clinical Immunology, vol. 177, pp. 12-17, 2017.

[73] J. Jahn, M. Spielau, C. Brandsch et al., "Decreased NK cell functions in obesity can be reactivated by fat mass reduction," Obesity (Silver Spring), vol. 23, no. 11, pp. 2233-2241, 2015.

[74] F. M. Wensveen, V. Jelenčić, S. Valentić et al., "NK cells link obesity-induced adipose stress to inflammation and insulin resistance," Nature Immunology, vol. 16, no. 4, pp. 376-385, 2015.

[75] S. Nishimura, I. Manabe, M. Nagasaki et al., "CD8 ${ }^{+}$effector T cells contribute to macrophage recruitment and adipose tissue inflammation in obesity," Nature Medicine, vol. 15, no. 8, pp. 914-920, 2009.

[76] B. C. Lee, M. S. Kim, M. Pae et al., "Adipose natural killer cells regulate adipose tissue macrophages to promote insulin resistance in obesity," Cell Metabolism, vol. 23, no. 4, pp. 685-698, 2016.

[77] T. E. O'Sullivan, M. Rapp, X. Fan et al., “Adipose-resident group 1 innate lymphoid cells promote obesity-associated insulin resistance," Immunity, vol. 45, no. 2, pp. 428-441, 2016.

[78] K. Wouters, K. Gaens, M. Bijnen et al., "Circulating classical

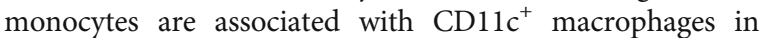
human visceral adipose tissue," Scientific Reports, vol. 7, no. 1, p. 42665, 2017.

[79] A. Shoae-Hassani, M. Behfar, S. A. Mortazavi-Tabatabaei, J. Ai, R. Mohseni, and A. A. Hamidieh, "Natural killer cells from the subcutaneous adipose tissue underexpress the NKp30 and NKp44 in obese persons and are less active against major histocompatibility complex class I nonexpressing neoplastic cells," Frontiers in Immunology, vol. 8, p. 1486, 2017.

[80] R. P. Donnelly, R. M. Loftus, S. E. Keating et al., "mTORC1dependent metabolic reprogramming is a prerequisite for NK cell effector function," Journal of immunology, vol. 193, no. 9, pp. 4477-4484, 2014.

[81] X. Michelet, L. Dyck, A. Hogan et al., "Metabolic reprogramming of natural killer cells in obesity limits antitumor responses," Nature Immunology, vol. 19, no. 12, pp. 13301340, 2018.

[82] O. Lamas, J. A. Martinez, and A. Marti, "Energy restriction restores the impaired immune response in overweight (cafeteria) rats," The Journal of Nutritional Biochemistry, vol. 15, no. 7, pp. 418-425, 2004.

[83] S. Perdu, B. Castellana, Y. Kim, K. Chan, L. DeLuca, and A. G. Beristain, "Maternal obesity drives functional alterations in uterine NK cells," JCI Insight, vol. 1, no. 11, article e85560, 2016.

[84] F. Mariani, P. Sena, G. Magnani et al., "PLZF expression during colorectal cancer development and in normal colorectal mucosa according to body size, as marker of colorectal cancer 
risk," ScientificWorld Journal, vol. 2013, article 630869, pp. 19, 2013.

[85] J. F. Clinthorne, E. Beli, D. M. Duriancik, and E. M. Gardner, "NK cell maturation and function in $\mathrm{C} 57 \mathrm{BL} / 6$ mice are altered by caloric restriction," Journal of Immunology, vol. 190, no. 2, pp. 712-722, 2013.

[86] C. B. Scanga, T. J. Verde, A. M. Paolone, R. E. Andersen, and T. A. Wadden, "Effects of weight loss and exercise training on natural killer cell activity in obese women," Medicine and Science in Sports and Exercise, vol. 30, no. 12, pp. 1666-1671, 1998.

[87] E. D. Shade, C. M. Ulrich, M. H. Wener et al., "Frequent intentional weight loss is associated with lower natural killer cell cytotoxicity in postmenopausal women: possible longterm immune effects," Journal of the American Dietetic Association, vol. 104, no. 6, pp. 903-912, 2004.

[88] C. M. Moulin, I. Marguti, J. P. S. Peron, A. Halpern, and L. V. Rizzo, "Bariatric surgery reverses natural killer (NK) cell activity and NK-related cytokine synthesis impairment induced by morbid obesity," Obesity Surgery, vol. 21, no. 1, pp. 112-118, 2011.

[89] D. R. Cottam, P. A. Schaefer, G. W. Shaftan, and L. D. G. Angus, "Dysfunctional immune-privilege in morbid obesity: implications and effect of gastric bypass surgery," Obesity Surgery, vol. 13, no. 1, pp. 49-57, 2003.

[90] M. H. Shin, J. Kim, S. A. Lim, J. Kim, S. J. Kim, and K. M. Lee, "NK cell-based immunotherapies in cancer," Immune network, vol. 20, no. 2, pp. e14-e14, 2020.

[91] V. Bachanova, S. Cooley, T. E. Defor et al., "Clearance of acute myeloid leukemia by haploidentical natural killer cells is improved using IL-2 diphtheria toxin fusion protein," Blood, vol. 123, no. 25, pp. 3855-3863, 2014.

[92] E. Liu, Y. Tong, G. Dotti et al., "Cord blood NK cells engineered to express IL-15 and a CD19-targeted CAR show long-term persistence and potent antitumor activity," Leukemia, vol. 32, no. 2, pp. 520-531, 2018.

[93] J. Cong, "Metabolism of natural killer cells and other innate lymphoid cells," Frontiers in Immunology, vol. 11, pp. 19891989, 2020.

[94] W. H. Qin, Z. S. Yang, M. Li et al., "High serum levels of cholesterol increase antitumor functions of nature killer cells and reduce growth of liver tumors in mice," Gastroenterology, vol. 158, no. 6, pp. 1713-1727, 2020.

[95] N. Assmann, K. L. O'Brien, R. P. Donnelly et al., "Srebp-controlled glucose metabolism is essential for NK cell functional responses," Nature Immunology, vol. 18, no. 11, pp. 11971206, 2017.

[96] M. Sukumar, J. Liu, Y. Ji et al., "Inhibiting glycolytic metabolism enhances CD8+ T cell memory and antitumor function," The Journal of Clinical Investigation, vol. 123, no. 10, pp. 4479-4488, 2013.

[97] E. R. Nelson, S. E. Wardell, J. S. Jasper et al., "27-Hydroxycholesterol links hypercholesterolemia and breast cancer pathophysiology," Science, vol. 342, no. 6162, pp. 1094-1098, 2013.

[98] Q. Wu, T. Ishikawa, R. Sirianni et al., "27-Hydroxycholesterol promotes cell-autonomous, ER-positive breast cancer growth," Cell Reports, vol. 5, no. 3, pp. 637-645, 2013.

[99] A. E. Baek, Y. R. A. Yu, S. He et al., "The cholesterol metabolite 27 hydroxycholesterol facilitates breast cancer metastasis through its actions on immune cells," Nature Communications, vol. 8, no. 1, p. 864, 2017.
[100] L. de la Cruz-Merino, N. Palazon-Carrion, F. HenaoCarrasco et al., "New horizons in breast cancer: the promise of immunotherapy," Clinical and Translational Oncology, vol. 21, no. 2, pp. 117-125, 2019.

[101] J. I. Luna, S. K. Grossenbacher, W. J. Murphy, and R. J. Canter, "Targeting cancer stem cells with natural killer cell immunotherapy," Expert Opinion on Biological Therapy, vol. 17, no. 3, pp. 313-324, 2017.

[102] E. Vivier, D. H. Raulet, A. Moretta et al., "Innate or adaptive immunity? The example of natural killer cells," Science, vol. 331, no. 6013, pp. 44-49, 2011. 